\title{
The Innovative Practice of Artificial Intelligence in the Inheritance of Chinese Xiangjin Art
}

\author{
Zhao Wenji $(\mathbb{D}$, Cui Rongrong $(\mathbb{D}$, and Niu Li $(\mathbb{D}$ \\ School of Design, Jiangsu Intangible Cultural Heritage Base, Jiangnan University, Wuxi 214122, China \\ Correspondence should be addressed to Zhao Wenji; 7180306008@stu.jiangnan.edu.cn
}

Received 14 December 2021; Accepted 3 January 2022; Published 17 January 2022

Academic Editor: Punit Gupta

Copyright (C) 2022 Zhao Wenji et al. This is an open access article distributed under the Creative Commons Attribution License, which permits unrestricted use, distribution, and reproduction in any medium, provided the original work is properly cited.

\begin{abstract}
This paper attempted to study the cultural characteristics and intelligent inheritance of Chinese Xiangjin brocade. Qualitative data was collected by in-depth interview method, and more than 1000 pieces of Xiangjin brocade of Suzhou Archives in China were measured for analyzing artistic features of their pattern design, color matching skills, and weaving technology, the study of which illustrated artistic features of modern Xiangjin brocade in detail and provides an important reference for its cultural and weaving inheritance. In addition, the artificial intelligence (AI) and the inheritance of Xiangjin art characteristics are integrated, the traditional handicrafts and digital art are docked, and the corresponding digital art protection mode is selected according to the characteristics of traditional handicrafts. The intelligent inheritance of cultural heritage not only changes the way of inheriting the artistic characteristics of Xiangin, but also changes people's general cognition of the cultural characteristics of Xiangjin. As a result, the integrity, authenticity, reliability, and comprehensiveness of the technological process of Xiangjin can be inherited, the patterns of Xiangjin can be preserved and applied, and the craft culture of Xiangjin can be inherited, protected, and developed.
\end{abstract}

\section{Introduction}

Xiangjin brocade is a kind of traditional Chinese silk-woven portraits and landscape paintings, whose carriers are screens, wall hangings, bookmarks, and calendars. Its main content is human image, landscape photography, Chinese painting, oil painting, calligraphy, commemorative activities, and poster art. Xiangjin brocade, as a valuable art work for collection, is mainly used as a souvenir for tourism, as an interior decoration, and as a gift for interpersonal communication and national exchanges. Due to its unique aesthetic value, Xiangjin brocade has been used as gifts for G20 leaders and soft decoration in the venue. Although European countries, Japan, and other countries started earlier in textile production, they did not form a large scale. With rich cultural deposits and superb traditional weaving technology, Chinese Xiangjin brocade has taken the lead and become a unique category of silk-weaving products, selling well in domestic and abroad markets for nearly a century. Arguably, Xiangjin brocade is not only the representative of innovative products in China's silk industry, but also a successful model of inheriting and developing Chinese silk culture.
The authors participated a cooperative project "Cataloguing and Studying of Xiangjin Brocade in Silk Archives of Suzhou, China" on December 1st, 2020, to January 15th, 2021. Altogether, the authors measured each Xiangjin brocade and catalogued more than 1000 pieces in Silk Archives of Suzhou from the period of The Republic of China, the founding of People's Republic of China, and opening and reform period. With the theme of inheriting silk culture and spreading silk art, the authors not only collected data on structural characteristics, drawing technique, size, color collocation, and area comparison of Xiangjin brocade, but also focused on the emergence, development, and technology in cataloging process.

Domestic researches on Xiangjin brocade are summarized as follows: (a) the introduction of Xiangjin brocade from the aspect of art and weaving skills. They are Yuan Xuan-ping's Inheritance and innovation of modern Chinese brocade art, Yuan Xuan-ping and Xu Zheng's Hangzhou weaved photograph, He Yunfei's The artistic features of Du Jinsheng brocade, Liu Kelong's master thesis The flower of oriental art-the art of Du Jinsheng brocade, and Peng Zeyi's 
History of handicraft industry in modern China (1840-1949). The making of Xiangjin brocade can be found in the third chapter of Peng's book, which involves the development of new products and the employment of new machine. The researchers mentioned above have studied the weaving process of Chinese brocade, Xiangjin brocade and West Lake brocade. (b) The introduction of Xiangjin brocade factories and Du Jinsheng brocade from the perspective of industrial development and management experience. They are represented by historical data for industry and commerce edited by Research Committee of Cultural and Historical Data of the CPPCC National Committee, Xie Mu and Wu Yongliang's China time-honored brand, Wang Xiang's The story of old brand, Yi Gan's Swaying ship: national industry and commerce (1900-1994), Li Gangyuan's An entrepreneur's innovative spirit and his cultural accumulation and world vision: A case analysis of Du Jinsheng and his Brand, and Hu Dan-ting's Survey of Hangzhou silk's reputation. Guo Xingmei takes brand as her research topic to scrutinize rough stages of introduction, digestion and innovation of modern Chinese silk technology. Taking three modern Chinese textile enterprise brands, i.e., "Shenxin Renzhong," "Meiya Silk," and "Wuhe Epai" as case examples, Guo explores how enterprises build up brand images and improve brand values by technology development, cultural exploration, and operation management. Then, Guo discusses the bottlenecks of contemporary textile enterprises and their brand development from the perspective of textile machinery innovation so as to offer some referential suggestions to the building of Chinese contemporary textile brands. (c) The introduction of Xiangjin brocade from the perspective of local history and brocade history. The representative works include Hangzhou in the Republic of China edited by Zhou Feng, Hangzhou local chronicles edited by Local Chorography of Hangzhou, New centennial chronicles of Zhejiang Province edited by Research Committee of Cultural and Historical Data of Zhejiang CPPCC, History of silk weaving industry in Jiangnan area in modern times edited by $\mathrm{Xu}$ Xinwu, and History of China modern textile edited by Editorial Committee of Modern Textile History of China. Besides, few scholars in other countries mentioned Chinese brocade when studying silk history, for example Japanese scholar Nuru Ono's Silk industry in Hangzhou. Although some Chinese scholars have introduced Du Jinsheng brocade from different perspectives, few of them combine Chinese brocade weaving techniques with modern textile design in practice. Other relevant researches can refer to the literature [1-5].

However, as the traditional brocade art design, Xiangjin brocade is attached to the specific cultural space and survival soil, is facing the plight of cultural disruption and lack of successors, and is gradually depressed and even going to decline. The inheritance of traditional Xiangjin brocade weaving skills is mainly family inheritance, industrialization inheritance, and so on. These inheritance methods are carried out under the relatively conservative and established space-asking mode, which is subject to many restrictions both in terms of the audience and the way of teaching. With the loss of time, it is not difficult to see that, due to the limited scope and space of inheritance and the excessive emphasis on specific groups, the traditional inheritance method has been difficult to maintain the survival of traditional crafts. Therefore, the selection of suitable technical means under the existing skill inheritance mode, especially the novel and unique artificial intelligence technology, can proceed from a unique artistic perspective. In order to develop a new concept of thinking to inherit, protect, and develop the traditional Xiangjin brocade skills. Since the advent of artificial intelligence, especially the wide application of "intelligent art," automatic art creation and performance appreciation have become a reality. It also has important practical application value in the fields of computer graphics, pattern design, light industry, and textile, as well as the inheritance of artistic features.

In order to protect this non-material cultural heritage, i.e., Chinese Xiangjin brocade, this paper adopts field survey method (visit Du Jinsheng Museum, Suzhou Chinese Silk Archives, and Xiangjin brocade enterprises) and market survey method to interpretate artistic characteristics of Chinese Xiangjin brocade in details, studies the application of Xiangjin brocade in modern textile design, especially in decorative crafts, and puts forward the suggestion of carrying on the intelligent inheritance of Xiangjin culture; that is, the artificial intelligence and the inheritance of Xiangjin art characteristics are integrated, the traditional handicrafts and digital art are docked, and the corresponding digital art protection mode is selected according to the characteristics of traditional handicrafts. The intelligent inheritance of cultural heritage not only changes the way of inheriting the artistic characteristics of Xiangjin, but also changes people's general cognition of the cultural characteristics of Xiangjin.

\section{Artistic Features of Chinese Xiangjin Brocade}

Chinese Xiangjin brocade has its unique artistic skills, such as artful color matching, delicate composition, vivid model, and profound meaning. It has beauty in both form and pursuit of elegant taste. It not only follows the scattered point penetration method of traditional literati paintings, but also draws on the western focus perspective method. In the long-term process of artistic development, it absorbs the excellent traditional elements of all periods, both ancient and modern time, China and foreign countries, developing its traditional and unique artistic features.

2.1. Exquisite Traditional Craftsmanship. As a pioneer of mechanized and large-scaled production in modern brocade industry in China, Xiangjin brocade took the lead in the innovation of traditional brocade in key processes such as craftsmanship, design and jacquard [6] and opened up a new era of brocade from manual weaving to industrial manufacture. It is of great significance in the history of Chinese silk weaving. Compared with paper products, it is tough and strong and more suitable to reveal the poetic charm of the original painting. Having artistic beauty and natural beauty, it is the perfect combination of painting, calligraphy art, and traditional brocade art. 
2.1.1. Colored Tapestry: Special Processing Technology. In the coloring process, the artisan workers use small script pens or eyebrow pens to paint different watercolors and art requirements on the black-white landscape brocade according to the picture's theme [7]. They should first have a handle on picture theme, coloring, small samples before coloring process, and then follow the principle of light color at first and deep after, far things at first and close things after, buildings at first and plants after. This can also be done flexibly according to the picture, that is, using less color to display painting content. In Figure 1, the coloring technique of the local pavilions built in Spring Dawn at Su Causeway of West Lake can make audience better appreciate the life-like scenery of lakes and hills. Compared with black-white Xiangjin brocade, the rich colors of the Imperial Monument Pavilion, lake, and boats break through the traditional planar layout expression, enabling audience to be more deeply impressed.

2.1.2. Multicolored Xiangjin Brocade: Perfect Technique. The coloration of multicolored Xiangjin brocade is mainly done through the ups and downs of the weft line. As a result, it is just like the photo itself, in which all the powder comes from the painter's hand. Though it keeps pace with Hunan brocade, the price is only the latter's one-twelfth, so it is cheap and cheerful. This kind of brocade is made of two groups of warps and multiple wefts interwoven into the multiple weft textile. One group of warps is the ground warp, while the other group is to press the floating weft tightly to connect the warp. In Figure 2, the multicolored Xiangjin brocade in the museum "Finch Plum" breaks through the traditional planar layout expression.

\subsubsection{Painting Xiangiin Brocade: Reproduction of} Masterpieces. Painting brocade is mainly woven with silk thread to manifest fine brushwork, freehand brushwork, and Chinese painting that combines fine brushwork with freehand brushwork. In this way, it has not only ink painting's quiet and elegant decorative effect, but also silk's natural and unrestrained charm. In addition to the use of color gradation to reveal shadow change, this multi-weft colorful Xiangjin brocade mainly uses the floating and sinking color of weft threads to form the color of the scene [8]. In Figure 3, the typical scenes that can best illustrate the heroine's personality in Zhaojun Goes Beyond the Great Wall as a Bride are meticulously colored to show the heroine's facial texture and emotional expression, including her clothing, hair on her temples, accessories, flowers, and pavilions.

2.1.4. Practical Xiangjin Brocade: Life-Oriented Technique. How to make Xiangjin brocade have both aesthetic taste and practical value? In order to increase the sales and practicability of silk-woven products, a practical Xiangjin brocade whose quality is far superior to that of Nanjing brocade was designed on the basis of multi-colored brocade.

It is the combination of painting, decoration, and art of living with character, landscape, and flowers as themes.

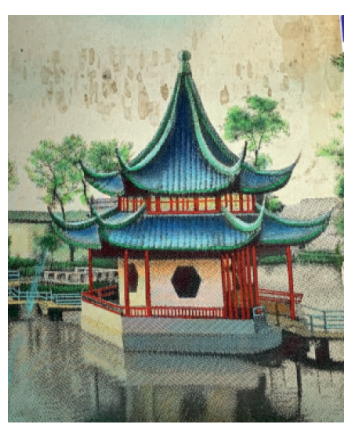

FIgURE 1: Colored Xiangjin brocade (archive collection, photos taken by the authors on Dec. $16^{\text {th }}, 2020$ ).

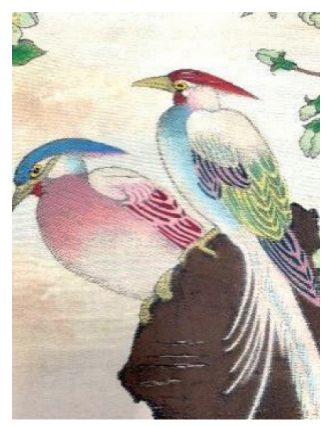

Figure 2: Multicolored Xiangjin brocade (archive collection, photos taken by the authors on Dec. $16^{\text {th }}, 2020$ ).

Compared with traditional brocade, the most striking feature of Xiangjin brocade is its life-oriented technique. The main use of traditional brocade like Sichuan brocade, Yun brocade, or Song brocade is for making clothes, while Xiangin brocade is mainly used for decoration from the date of its birth. As West Lake Expo Daily introduced, "Xiangjin brocade can be used for hall hanging, study ornament, orchid boudoir enjoyment, elegant and pleasant" [9]. Business Monthly also reported, "one important art invention which can be used for gift-giving and decoration." The two-warp-multi-weft structure of practical brocade becomes one-warp-three-weft structure, in which one group of warps interweave weft to form ground tissue, while another two groups of wefts weave flowers on ground tissue. Practical brocade includes silk fan, back cushion, bedspread, and handbags. Handbags are made of fabric scraps of raw materials so they can save cost and increase output, rather popular with customers at home and abroad. It is a true novelty in silk industry. In Figure 4, it is a Xiangjin brocade made from the Western classic drawing "reading" for hanging in bedroom.

2.2. Elegant Color: Affection for the East. Inheriting the main colors of Chinese traditional brocade, Xiangjin brocade is often colored with warm colors like red, yellow, and green. Unlike quiet, elegant, and simple style of Chinese traditional brocade, it uses gorgeous, rich, and chunky colors to reflect its strong and unrestrained artistic features. Regarding color matching skills, it employs the traditional "color change" 


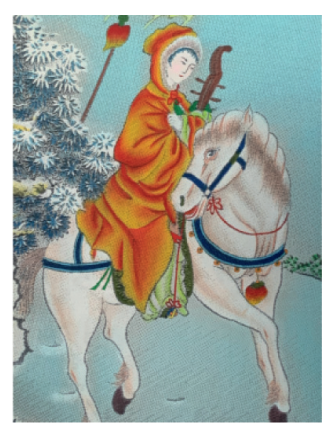

FIgUre 3: Painting Xiangjin brocade (archive collection, photos taken by the authors on Dec. $\left.16^{\text {th }}, 2020\right)$.

skills to make the picture natural, harmonious, and unified, while reducing the visual stimulation that chunky color produces. In Figures 5 and 6, "Comprehensive Painting Indicating Five Norms" that is themed with two gorgeous peacocks and a pair of cranes is the most representative. On the right, the green leaves and wild pines match with white willow warbler, while on the lower left, there is a pair of intimate mandarin ducks. Rich warm colors like red, yellow, and green match with black, white, and gray, which present a vibrant, lively, lovely, and harmonious decorative effect.

\subsubsection{Sights of the West Lake Copied Vividly on Xiangjin} Brocade. The well-known folk artist Zhang Daoyi mentioned in his work Folk Art in China, "Characterized by diversity, practicality and appreciation, Chinese folk arts have a strong local color" [10]. Japanese technologist Yanagi Souetsu argued, "All the handicrafts in history that are closely related to local history and culture have striking local features" [11]. Chinese Xiangjin brocade is indigenous to Hangzhou, and West Lake Xiangjin brocade is the most typical one, so it has distinct cultural characteristics of the West Lake. Xiangin brocade takes photos as negatives and absorbs the strong points of photographic art through the form of brocade paintings. Thus, it has multiple styles of traditional national painting, western photographic art, and modern oil painting. Its silk weaving paintings with the same effect as photos are exquisite, vivid, and lifelike, so it blends inheritance with innovation.

2.2.2. Profound Implications of Oriental Charm. K'o-ssu, embroidery craft, and double-weft brocade craft are often utilized to weave silk articles based on Chinese paintings and calligraphic works. With the development of pattern jacquard technology, Xiangjin brocade employs different colors and weaving points with brightness to represent traditional Chinese oriental element; thus the color change becomes soft. Figures 7 and 8 are part of yarn-dyed and color-filled silk Chinese painting "Wu Guandai's Landscape Painting" $(27 \times 98 \mathrm{~cm})$. They have the following features. First, black, white, and light-yellow rayon are utilized to treat weft color. Triple-weft structure which is more complex than the blackwhite double weft is employed to the weave the textile. Second, color filling method is used on the basis of yarn-

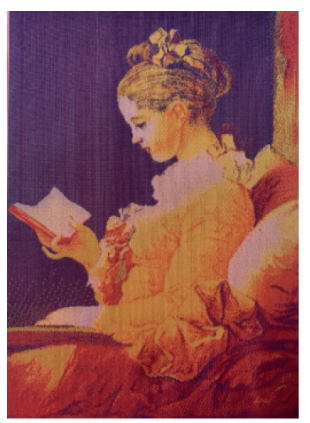

FIgUre 4: Practical Xiangjin brocade (archive collection, photos taken by the authors on Dec. $16^{\text {th }}, 2020$ ).

dyed knitting. For example, painted color techniques are employed to make red, yellow, blue, green, and ochre on the picture. Third, it is very rare that Du Jinsheng trademark, factory's name, and four Chinese characters "Wu Cai Jin Xiu" are weaved on the bottom of the picture at the same time. Fourth, this work is rather grand, whose weft width is $27 \mathrm{~cm}$, the number of jacquard needles is more than 2000, and the number of grain plates is more than 8000 . This technology took the lead in the 1930s, which made near objects and distant landscape in the painting have rich levels, trees, houses and figures have strong three-dimensional sense, fully demonstrating the unique taste of silk paintings of landscape and figures.

2.2.3. Multivariate Utility of Intrinsic Value. As an important member of folk art, the themes of Xiangjin brocade are mostly derived from real life, which truly record the West Lake landscape culture, garden culture, architecture culture, folk culture, and religious culture, helping later generations intuitively appreciate scenery of the past. The themes include moral customs, humanistic education, and psychological concepts. Since its birth, a series of works such as Xiangjin brocade cushion, hanging scroll, wall calendar, and table cloth with great spirits has been innovated. In Figures 9 and 10 , accompanied by a red maid, the legendary Goddess Avalokitesvara stands bare-footed on the lotus stage. She dresses in white clothes, smiling to an innocent infant beside her. This brocade shows people's high respect for Avalokitesvara, expressing Chinese nation's strong wish for reproducing offspring; thus it has a good educational effect.

\subsection{Innovative Practice of Xiangjin Brocade in Modern Textile} Design: Take Du Jinsheng Brocade as an Example. Chinese Xiangjin brocade is not only a product, but also a work of art, representing the peak of the development of contemporary Chinese brocade. With regard to brocade objects, there are not only fabric pattern paintings, but also large-scale paintings, which harmoniously display flat and three-dimensional paintings, especially vividly record different historical and cultural forms. In terms of painting performance, it inherits techniques of traditional Chinese paintings, religion paintings, New Year pictures, print makings, classical oil paintings, and western contemporary paintings. 


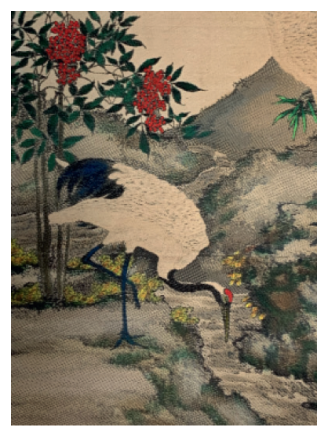

FIGURE 5: Crane in comprehensive painting indicating five norms (archive collection, photos taken by the authors on Jan. $6^{\text {th }}, 2021$ ).

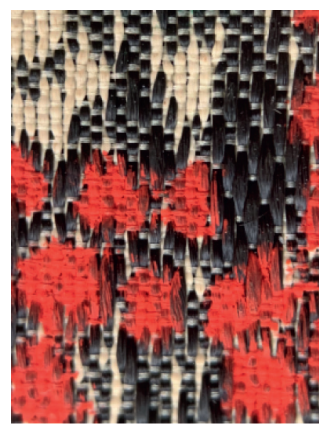

Figure 6: Hand coloring of comprehensive painting indicating five norms (archive collection, photos taken by the authors on Jan. $6^{\text {th }}$, 2021).

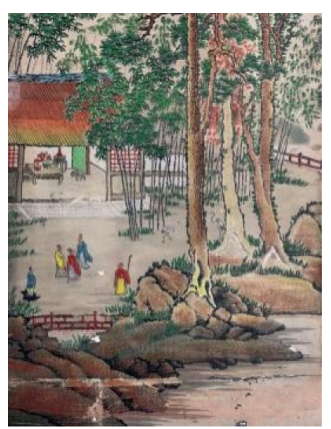

FIgURE 7: Part of Wu Guandai's landscape painting (archive collection, photos taken by the authors on Jan. $6^{\text {th }}, 2021$ ).

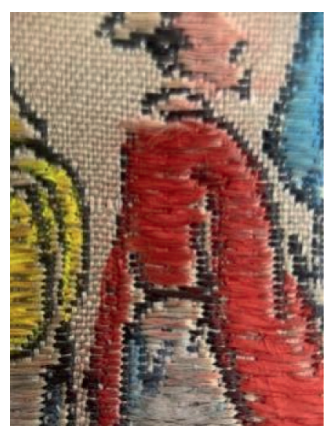

FIgURE 8: Hand coloring of Wu Guandai's landscape painting (archive collection, photos taken by the authors on Jan. $6^{\text {th }}, 2021$ ).
Its artistry exceeds the most precious "double brocade" hanging scroll in Ming and Qing dynasties. In the aspect of decorative brocade for daily necessities, rayon square table blanket, rayon round table blanket, and broad blessing table blanket have been successively produced.

2.3.1. Social Investigation of Innovative Practice of Xiangjin Brocade. In recent decades, there are different kinds of Xiangjin brocade products with different prices. To know the present situation of Xiangjin brocade in Chinese market, the authors used "Xiangjin brocade," "brocade," "traditional handicraft," "the intangible cultural heritage," "traditional textiles," "China's time-honored brand," and "Du Jinsheng" as key words to search information of Xiangjin brocade on three Chinese online platforms "Taobao," "Joy Buy," and "Tmall." The information included brocade brand, style, price, category, comment, and target population. Altogether, 1300 pictures were screened out.

Then, 13 famous brands of silk industry in Chinese market were selected, whose information was compared with that of products sold on the Internet to find out the dissimilarities between the two. Orange software was employed to analyze the collected data and position the present situation and dilemma of Chinese Xiangjin brocade.

Third, a survey was made on which kind of Xiangjin brocade customers prefer through closed Interview questions.

It can be seen from Table 1 and Figure 10 that more than half of the participants know something about the technique of Chinese Xiangjin brocade. Although online sales are booming at present, branded Xiangjin brocade enterprises are accepted by most consumers, for they can better represent Chinese brocade culture. More than half of the consumers do not accept the high price of Xiangjin brocade. Most interviewees pay more attention to the branded Chinese brocade handicrafts, including HSDP, Wensli, Hangsilian, Fuhua, Hongluo, and Jinyuan, among which Du Jinsheng brocade is the most popular one.

2.3.2. Innovative Practice of Du Jinsheng Brocade. As the representative of Hangzhou brocade, Du Jinsheng brocade came into being in 1921, including Xiangjin brocade, decorative brocade, and brocade for clothing whose more than 1640 varieties have become China's representative brocade [12]. According to different customers, traditional theme was made into patterns of different levels through mud floor, skimming silk, arrangement shadiness, and side shadiness. With regard to manifestation, free and irregular composition have replaced the traditional balanced and single composition. Du Jinsheng brocade have made three biggest textile works, i.e., hanging Xiangjin brocade, Xiangjin brocade carpet, landscape Xiangjin brocade. Among them, the Xiangjin brocade Ten Thousand $\mathrm{Li}$ of Rivers and Mountains $(42 \times 1125 \mathrm{~cm})$ is known as the longest silk weaving picture in the world. One of them was hung in the hall of Swedish parliament in Stockholm.

Paying much attention to the market, Du Jinsheng brocade not only produced blanket and back cushion, but 

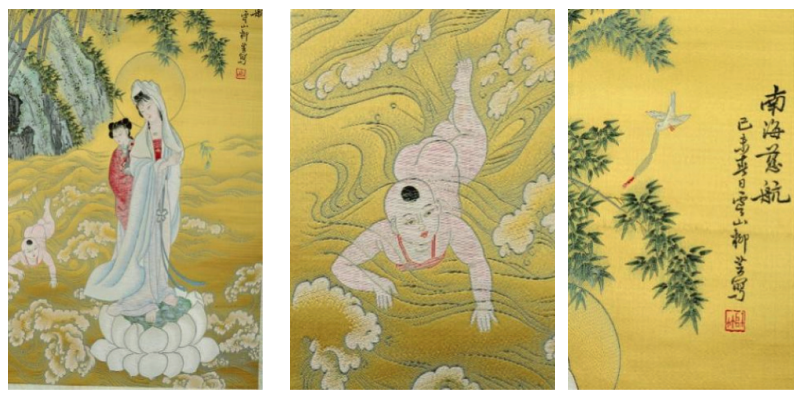

Figure 9: Hanging scroll South China Sea Avalokitesvara (collection in Du Jinsheng Museu, photos taken by the authors on Jan. $\left.5^{\text {th }}, 2021\right)$.

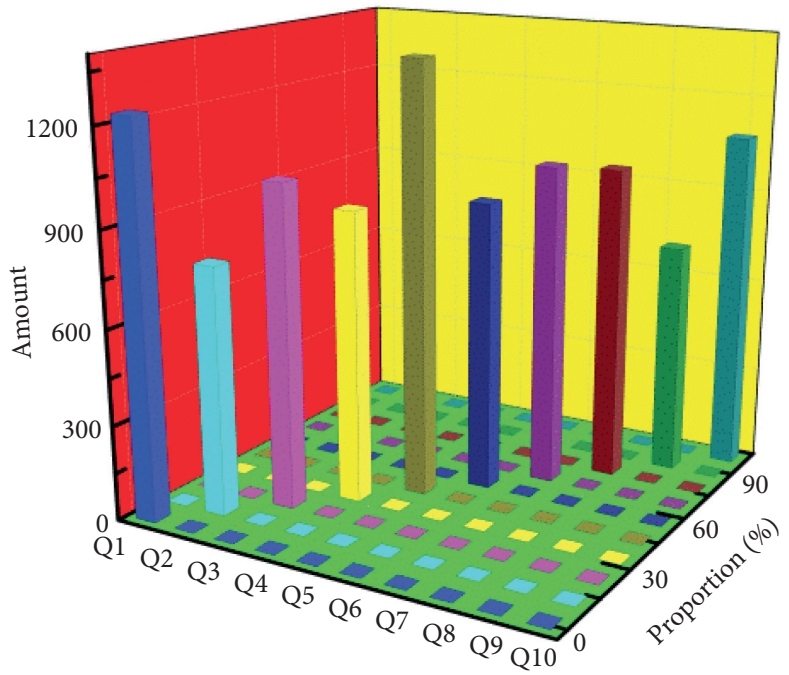

FIGURE 10: Questionnaire results.

TABLE 1: Interview questions.

\begin{tabular}{|c|c|c|c|c|}
\hline No. & Interview questions & Choices $(\mathrm{Y} / \mathrm{N})$ & Amount & $\%$ \\
\hline \multirow{2}{*}{1} & \multirow{2}{*}{ Do you know the technique of Chinese Xiangjin brocade? } & $\mathrm{Y}$ & 1233 & 61.65 \\
\hline & & $\mathrm{N}$ & 767 & 38.35 \\
\hline \multirow{2}{*}{2} & \multirow{2}{*}{ Can you give two artistic features of Xiangjin brocade? } & $\mathrm{Y}$ & 784 & 39.20 \\
\hline & & $\mathrm{N}$ & 1216 & 60.80 \\
\hline \multirow{2}{*}{3} & \multirow{2}{*}{ Do you think Xiangjin brocade can be applied in modern society? } & $\mathrm{Y}$ & 1021 & 51.05 \\
\hline & & $\mathrm{N}$ & 979 & 48.95 \\
\hline \multirow{2}{*}{4} & \multirow{2}{*}{ Do you anticipate the application of Xiangjin brocade in modern textile design? } & $\mathrm{Y}$ & 923 & 46.15 \\
\hline & & $\mathrm{N}$ & 1077 & 53.85 \\
\hline \multirow{2}{*}{5} & \multirow{2}{*}{ Do you pay more attention to Xiangjin brocade with a famous brand? } & $\mathrm{Y}$ & 1364 & 68.20 \\
\hline & & $\mathrm{N}$ & 636 & 31.80 \\
\hline \multirow{2}{*}{6} & \multirow{2}{*}{ Can you accept Xiangjin brocade with a price of $¥ 1000-2000 ?$} & $\mathrm{Y}$ & 921 & 46.05 \\
\hline & & $\mathrm{N}$ & 1079 & 53.95 \\
\hline \multirow{2}{*}{7} & \multirow{2}{*}{ Do you know some brands of Chinese Xiangjin brocade? } & $\mathrm{Y}$ & 1012 & 50.60 \\
\hline & & $\mathrm{N}$ & 988 & 49.40 \\
\hline \multirow{2}{*}{8} & \multirow{2}{*}{ Do you like some innovative design of Chinese Xiangjin brocade? } & $\mathrm{Y}$ & 1001 & 50.05 \\
\hline & & $\mathrm{N}$ & 998 & 49.95 \\
\hline \multirow{2}{*}{9} & \multirow{2}{*}{ Do you think branded Xiangjin brocade can represent Chinese textile culture? } & $\mathrm{Y}$ & 738 & 36.90 \\
\hline & & $\mathrm{N}$ & 1262 & 63.10 \\
\hline \multirow{2}{*}{10} & \multirow{2}{*}{ Do you think Chinese traditional textile industry need some improvement? } & $\mathrm{Y}$ & 1078 & 53.90 \\
\hline & & $\mathrm{N}$ & 922 & 46.10 \\
\hline
\end{tabular}

also designed Xiangjin brocade bedspread and silk-wool curtain, which are washable and bright colored and have strong senses of relief, so it has both practical value and ornamental value [13]. The brocade silk bags made in China that ladies of various countries held in G20 Hangzhou Summit can be regarded as the most representative and influential innovative works of $\mathrm{Du}$ Jinsheng brocade (see Figure 11). The inspiration came from glacier frostwork 
whose beautiful lines indicate the fantastic dreamy scenery of nature. Against elegant purple, the frostworks with conch divergent crystalline lines are pure and clear, classic and fashionable, and very pleasing to the eye. Theme pattern "fantastic frostwork" employs modern positioning yarndyed jacquard technology, natural reactive dyes, platform floral printings, biological enzyme finishing technology to make its color uniformly bright. Silk bags match with perfect leather piping, grand and elegant, exactly reflecting the excellent quality of national gifts. Figure 12 shows $\mathrm{Du}$ Jinsheng brocade back cushion that is themed with the Bronze Sparrow Terrace.

Meanwhile, through collation and analysis, we can classify more than 1000 Xiangjin brocade fabrics collected from Suzhou Archives in China; that is, they can be divided into four categories: landscape brocade, figure image brocade, painting brocade, and practical brocade from different perspectives of fabric theme, expression method, organizational structure, and use function. Furthermore, through the above analysis, we can restore the artistic characteristics and timeliness of the brocade industry in different periods and take history as a mirror to examine its value and significance to the contemporary textile industry and product design.

\section{Results and Discussion}

From the above statement, we have collected and sorted out the technological process, the implication of the pattern, and the cultural connotation of the Xiangjin brocade and deeply analyzed the artistic design, customs, and religious beliefs reflected behind the elephant of the Xiangjin brocade. And through the comparative study of the brocade ways of different regions and different cultures in the same period, taking the technological characteristics of Xiangjin brocade as a starting point, this paper summarizes the existing inheritance problems of Xiangjin brocade craftsmanship.

The inheritance of traditional Xiangjin brocade skills mainly includes family inheritance, industrialization inheritance, and so on, but with the passage of time, due to the limited scope and space of inheritance, too much emphasis on specific groups, the traditional way of inheritance has been difficult to maintain the survival of traditional technology. Therefore, the selection of appropriate technical means under the existing skill inheritance mode, especially the novel and unique artificial intelligence technology, can form a new idea to inherit, protect, and develop the traditional Xiangjin brocade skills. With regard to the application of artificial intelligence technology in the inheritance of cultural heritage, there are already some achievements, such as the literature [14-16].

3.1. Segmentation of Xiangjin Brocade Image Based on DeepLabv3+. Image semantic segmentation is a technology that enables computers to understand images, and it can realize object category recognition and high-precision image segmentation simultaneously in a network. In recent years, this technology has achieved remarkable results in the semantic segmentation of visible images [17-19], while the research on the scene understanding of Xiangjin brocade images is still in the blank stage. In this paper, the Xiangjin brocade image of Suzhou Archives is used as the training sample, and DeepLabv3+ network is used as the training model [20]. In order to improve the precision of final segmentation, the whole image of Xiangjin brocade was segmented from the complex background, and the semantic segmentation results were postprocessed by combining image morphology method. At the same time, it also lays a foundation for finding regions of interest, feature extraction, and state classification.

With the advent of full convolutional networks (FCN), Long [21] et al. replaced the full connection layer in mainstream classification networks such as AlexNet, VGGNet, and GoogLeNet into the convolutional layer. Finally, transpose convolution is added to restore the feature graph to its original size [22], so that the spatial location features of image pixels can be retained and semantic segmentation has a breakthrough development. In this paper, DeepLabv3+, which has the highest score in MIoU tests in public data sets in recent years, is used as a network to train the semantic segmentation model of images of Xiangjin brocade. The image segmentation results of Figures 2 and 3 are obtained in Figures 13 and 14. Figures 13 and 14 show the exploration of artificial intelligence technology in the cultural inheritance of Xiangjin brocade fabrics. Their significance lies in the more detailed analysis and inheritance of Xiangin brocade images in the form of digital pictures. It allows people to segment the whole Xiangjin image from the complex background, so as to improve the final segmentation accuracy, narrow the target object analysis scope for searching the region of interest, and carry out feature extraction and state classification.

\subsection{Intelligent Inheritance of Cultural Characteristics of} Chinese Xiangjin Brocade. The application of artificial intelligence can expand the channels of inheriting the artistic characteristics of Xiangjin, from the previous family inheritance and industrialization inheritance to the current digital, VR image preservation, and other ways, so that the cultural heritage like Xiangjin, which is limited by time and space and is not easy to inherit, has been caught on the express train of "the development of the times." Specifically, the existing Xiangjin brocade fabrics can be digitally preserved and a digital museum of Xiangiin brocade can be established. Even if the years wear out the real thing, people can learn the whole picture of Xiangin brocade fabric with the help of digital platform. Specifically, the 360-degree panoramic VR technology can be used to record the skills of the current inheritors of the brocade through audio, video, and animation. Through stories like those told in Xiangjin brocade and VR graphic tutorials, people can immerse themselves in the virtual world across time and space. The teaching of VR techniques can make the successors learn systematically and make them master the skills of Xiangjin brocade knitting skillfully. VR pattern tutorials can also enable successors to learn the pattern tutorials left by the older generation of tapestry masters through pattern 

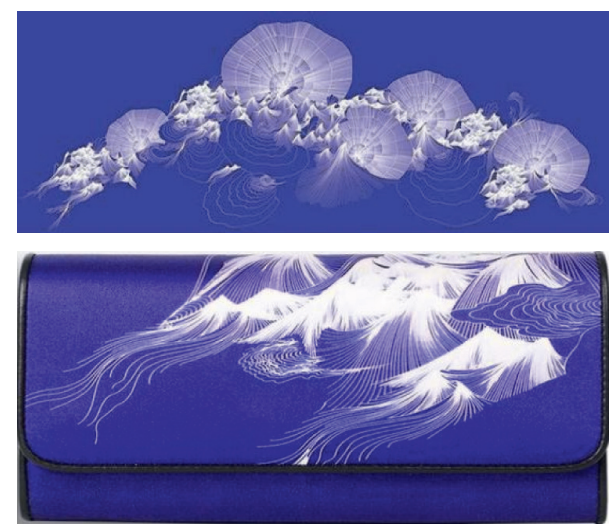

Figure 11: Pattern of fantastic frostwork Xiangjin brocade handbag and its front.
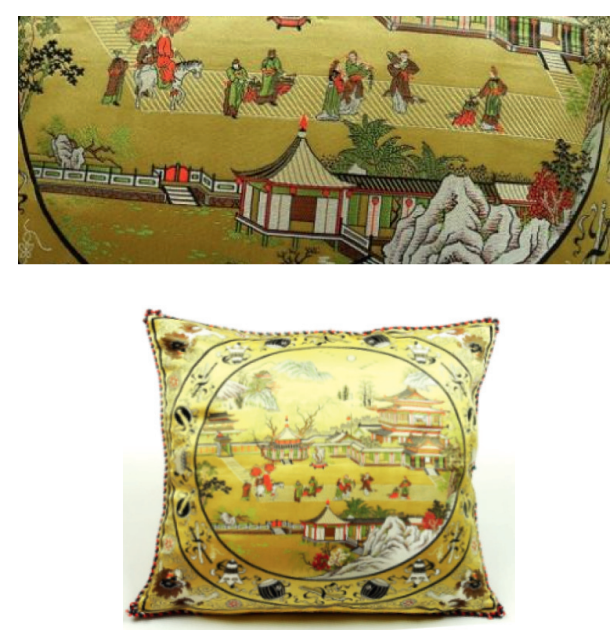

Figure 12: Pattern of bronze sparrow terrace Xiangjin brocade back cushion and its front.

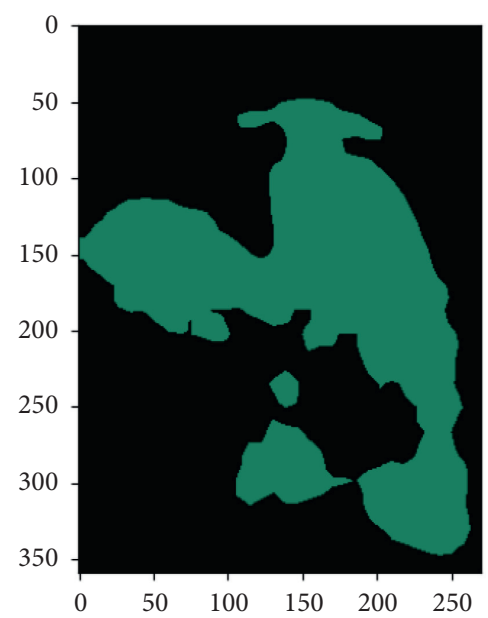

Figure 13: Segmentation diagram of DeepLabv3+ in Figure 2.

classification, so as to avoid the loss of skills as much as possible. For another example, we can use AR technology to develop a Xiangjin brocade experience game like "Happy Farm." In the web page promotion of Xiangjin brocade, scenes such as

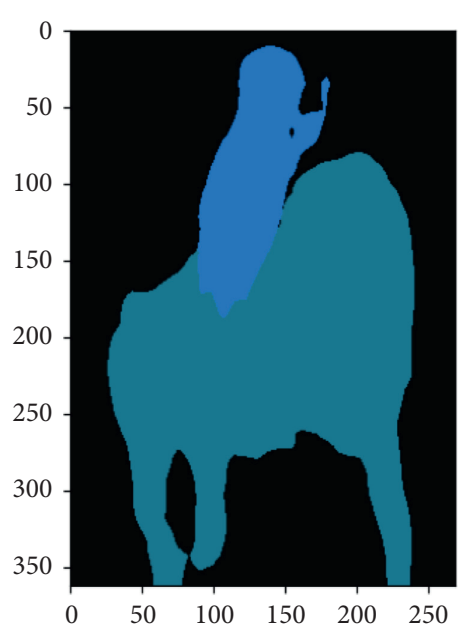

Figure 14: Segmentation diagram of DeepLabv3+ in Figure 3.

planting mulberry, rewinding, knitting, and packing are added to let the experiencers make guided virtual production through Mini Program. Then, the background will record the user's entire production process and make it manually, which can be sent home by express delivery after the user has paid for it. In this way, users can not only experience the charm of traditional Xiangjin culture, but also learn the production process of Xiangjin, which is extremely beneficial to the inheritance of Xiangjin culture. Artificial intelligence makes people know more about Xiangjin culture through sensory interaction, which not only enhances people's immersive experience, but also effectively makes up for the lack of communication between the two sides. Therefore, the integrated development of VR technology and the inheritance of the characteristics of Xiangjin brocade art not only changes the way of inheritance of the characteristics of brocade art, but also changes people's general cognition of the characteristics of brocade culture. In addition, the methods of machine learning and in-depth learning can also be applied to the inheritance of the artistic characteristics of Xiangjin brocade. There are some relevant literature as [23-29]. The comprehensive application of the two fully reflects the role of innovation in inheriting traditional culture such as Xiangin brocade, so that the integrity, authenticity, reliability, and comprehensiveness of the technological process of Xiangjin brocade can be inherited, and the patterns of Chinese Xiangjin brocade can be preserved and applied.

In short, combined with literature and image data, this paper investigates the evolution of Xiangjin brocade and the changes of silk culture on the basis of regional characteristics. It is of great practical significance to systematically and scientifically research and study the different development periods of Xiangjin brocade.

\section{Conclusion}

Chinese Xiangjin brocade draws on the essence of Sichuan brocade, Yun brocade, and Song brocade and also dissolves the wit and beauty of Hangzhou brocade at the same time, so it forms its own unique artistic style, well-known as "the 
flower of oriental art." In this paper, we summarized and analyzed the artistic modeling characteristics of Chinese Xiangjin brocade and demonstrated that the aesthetics contained in Xiangjin brocade is consistent with the contemporary public way of life and summarized and analyzed the inherent deficiency of Chinese Xiangin brocade in inheritance. Therefore, in order to make the process of Xiangjin brocade complete, real, reliable, and comprehensive to inherit, and the pattern of Xiangjin can be preserved and applied, we should adopt the new technology of artificial intelligence. This can not only change the way of inheriting the artistic characteristics of Xiangjin, but also change people's general cognition of the cultural characteristics of Xiangjin. The comprehensive application of the two can fully reflect the role of innovation in inheriting traditional culture like Chinese Xiangjin brocade. However, due to the regional limitations of sample collection, the scope of application of the conclusions is limited. At the same time, the technology of artificial intelligence is also developing rapidly, such as machine learning methods and deep learning methods, which can become new tools for us to study the cultural heritage of Chinese Xiangjin brocade.

\section{Data Availability}

The datasets used and/or analyzed during the current study are available from the corresponding author on reasonable request.

\section{Conflicts of Interest}

The authors declare no potential conflicts of interest with respect to the research, authorship, and/or publication of this article.

\section{Acknowledgments}

The authors acknowledge the Key Project of Philosophy and Social Science Research in Colleges and Universities (2018SJZDA130) and the "333 Project" of Jiangsu Province for Young and Middle-aged Leading Talents at the Second Level (BRA2016366).

\section{References}

[1] R. A. Bentley and M. J. O’Brien, The Acceleration of Cultural Change: From Ancestors to Algorithms, MIT Press, Cambridge, MA, USA, 2017.

[2] K. Chen, D. Lu, Z. Jin, M. Su, and J. Jin, "Song brocade in the ming and qing dynasties," Clothing and Textiles Research Journal, vol. 38, no. 4, pp. 285-297, 2020.

[3] A. Filomeno, "Application of the western art form based on artificial intelligence," Cultural Communication And Socialization Journal, vol. 1, no. 1, pp. 16-17, 2020.

[4] Z. Hongxia and L. Y. U. Zhijia, "Textiles design idea based on transboundary reference," Cotton Textile Technology, vol. 49, no. 597, 2021.

[5] X. Hu, "Usability evaluation of E-Dunhuang cultural heritage digital library," Data and Information Management, vol. 2, no. 2, pp. 57-69, 2018.
[6] N. Ibrahim and N. M. Ali, "A conceptual framework for designing virtual heritage environment for cultural learning," Journal on Computing and Cultural Heritage, vol. 11, no. 2, pp. 1-27, 2018.

[7] H. Kuang and J. Wu, "Survey of image semantic segmentation based on deep learning," Computer Engineering and Applications, vol. 55, no. 19, pp. 12-21, 2019.

[8] C. Li, Du Jinsheng Brocade, Donghua University Press, Shanghai, China, 2009.

[9] G. Li, The Silk King Du Jinsheng, Tianjin People's Publishing House, Tianjin, China, 2011.

[10] S. Li and Z. Wang, "Mural art in the context of new media: a new "crossover" of mural art driven by artificial intelligence," in Proceedings of the 2nd International Conference on Language, Art and Cultural Exchange (ICLACE 2021), pp. 530-533, Atlantis Press, Dali, China, January 2021.

[11] K. Liu, The Flower of Oriental Art-The Art of Du Jinsheng Brocade, Hangzhou Normal University, Zhejiang, China, 2011.

[12] X. Liu, "Artistic reflection on artificial intelligence digital painting," Journal of Physics: Conference Series. IOP Publishing, vol. 1648, no. 3, Article ID 032125, 2020.

[13] J. Long, E. Shelhamer, and T. Darrell, "Fully convolutional net-works for semantic segmentation," in Proceedings of the IEEE Conference on Computer Vision and Pattern RecoRuiriou, pp. 3431-3440, Boston, MA, USA, June 2015.

[14] C. Lv, Hangzhou Time-Honored Brand, Hangzhou Publishing House, Hangzhou, China, 1998.

[15] S. Minaee, Y. Y. Boykov, F. Porikli, A. Plaza, N. Kehtarnavaz, and D. Terzopoulos, "Image segmentation using deep learning: a survey," IEEE Transactions on Pattern Analysis and Machine Intelligence, 2021.

[16] R. Pierdicca, M. Paolanti, F. Matrone et al., "Point cloud semantic segmentation using a deep learning framework for cultural heritage," Remote Sensing, vol. 12, no. 6, p. 1005, 2020.

[17] F. G. Rivera, E. Brolin, A. Syberfeldt, D. Högberg, A. Iriondo, and E. P. Luque, "Using virtual reality and smart textiles to assess the design of workstations," in Proceedings of the SPS2020: Swedish Production Symposium, vol. 13, p. 145, IOS Press, Jönköping, Sweden, October 2020.

[18] J. Tan, L. Shao, N. Y. K. Lam, A. Toomey, and L. Ge, "Intelligent textiles: designing a gesture-controlled illuminated textile based on computer vision," Textile Research Journal, 2021.

[19] S.-K. Tan, H.-H. Lim, S.-H. Tan, and Y.-S. Kok, “A cultural creativity framework for the sustainability of intangible cultural heritage," Journal of Hospitality \& Tourism Research, vol. 44, no. 3, pp. 439-471, 2020.

[20] S.-K. Tan, S.-H. Tan, Y.-S. Kok, and S.-W. Choon, "Sense of place and sustainability of intangible cultural heritage - the case of George Town and Melaka," Tourism Management, vol. 67, pp. 376-387, 2018.

[21] C. Wang, Research on Infrared Image Semantic Segmentation Technology Based on Deep learning, University of Chinese Academy of Sciences (Shanghai Institute of Technical Physics, Chinese Academy of Sciences), Shanghai, China, 2017.

[22] West Lake Expo Ed, "West lake expo daily," 1929.

[23] Y. Xiao and J. Hu, "The inheritance and spreading of confucianism in modern China and South Korea," in Proceedings of the 2019 5th International Conference on Social Science and Higher Education (ICSSHE 2019), pp. 1061-1064, Atlantis Press, Xiamen, China, January 2019.

[24] S. Yanagi, Handicraft Culture, Guangxi Normal University Press, Guangxi, China, 2006. 
[25] M. Yuan, H. Huang, and C. Zhou, "Research progress of image semantic segmentation based on fully supervised learning," Computer Engineering and Applications, vol. 57, no. 4, pp. 43-54, 2021.

[26] N. Yun, "Research on manufacturing technology and cultural connotation of guangxi rongshui miao brocade strap," Journal of Sociology and Ethnology, vol. 3, no. 1, pp. 18-22, 2021.

[27] D. Zhang, Zhang Daoyi's Comments on Folk Arts, Shandong Fine Arts Publishing House, Shandong, China, 2008.

[28] J. Zhu, The History of Du Jinsheng Brocade, p. 36, China Social Sciences Publishing House, Beijing, China, 2014.

[29] W. Zhang, J. Pang, K. Chen, and C. C Loy, "K-net: towards unified image segmentation," Advances in Neural Information Processing Systems, p. 34, 2021, https://arxiv.org/abs/2106. 14855. 